\title{
Apigenin inhibits UVA-induced cytotoxicity in vitro and prevents signs of skin aging in vivo
}

\author{
SUNGJIN CHOI $^{1 *}$, JEUNGYEUN YOUN ${ }^{1 *}$, KARAM KIM $^{1}$, DA HYE JOO $^{1}$, SHANGHUN SHIN $^{1}$, \\ JEONGJU LEE ${ }^{1}$, HYUN KYUNG LEE ${ }^{1}$, IN-SOOK AN ${ }^{2}$, SEUNGBIN KWON ${ }^{2}$, HAE JEONG YOUN ${ }^{3}$, \\ KYU JOONG AHN ${ }^{3}$, SUNGKWAN AN ${ }^{1}$ and HWA JUN CHA ${ }^{1}$ \\ ${ }^{1}$ Korea Institute for Skin and Clinical Sciences, Konkuk University, Seoul 143-701; \\ ${ }^{2}$ GeneCellPharm Incorporated, Venture Center II, Cheongju-si, Chungcheongbuk-do 361-951; \\ ${ }^{3}$ Department of Dermatology, Konkuk University School of Medicine, Seoul 143-701, Republic of Korea
}

Received October 7, 2015; Accepted May 25, 2016

DOI: $10.3892 / \mathrm{ijmm} .2016 .2626$

\begin{abstract}
Apigenin (4',5,7-trihydroxyflavone) is a flavone that has been reported to have anti-inflammatory, antioxidant and anti-carcinogenic properties. In this study, we investigated the protective effects of apigenin on skin and found that, in experiments using cells, apigenin restored the viability of normal human dermal fibroblasts (nHDFs), which had been decreased by exposure to ultraviolet (UV) radiation in the UVA range. Using a senescence-associated (SA)- $\beta$-gal assay, we also demonstrate that apigenin protects against the UVA-induced senescence of nHDFs. Furthermore, we found that apigenin decreased the expression of the collagenase, matrix metalloproteinase (MMP)-1, in UVA-irradiated nHDFs. UVA, which has been previously identified as a photoaging-inducing factor, has been shown to induce MMP-1 expression. The elevated expression of MMP-1 impairs the collagen matrix, leading to the loss of elasticity and skin dryness. Therefore, we examined the clinical efficacy of apigenin on aged skin, using an apigenin-containing cream for clinical application. Specifically, we measured dermal density, skin elasticity and the length of fine wrinkles in subjects treated with apigenin cream or the control cream without apigenin. Additionally, we investigated the effects of the apigenin-containing cream on skin texture, moisture and transepidermal water loss (TEWL). From these experiments, we found that the apigenin-containing cream increased dermal density and elasticity, and reduced fine wrinkle length. It also improved skin evenness, moisture content and TEWL. These results clearly demonstrate the biological effects of apigenin, demonstrating both its cellular
\end{abstract}

Correspondence to: Dr Hwa Jun Cha, Korea Institute for Skin and Clinical Sciences, Konkuk University, 120 Neungdong-ro, Gwangjin-gu, Seoul 143-701, Republic of Korea

E-mail: hjcha@konkuk.ac.kr

${ }^{*}$ Contributed equally

Key words: apigenin, ultraviolet A, fibroblast, matrix metalloproteinase, skin, wrinkle, aging, barrier and clinical efficacy, and suggest that this compound holds promise as an anti-aging cosmetic ingredient.

\section{Introduction}

Aging is defined as the progressive accumulation of damage over time, leading to the disruption of functions at the cellular, tissue and organ levels. Eventually, disease and death are induced by a complex, multifactorial process, involving genetic, endogenous and environmental factors (1). The consequences of human aging are mostly visible in the skin, manifesting as increased wrinkling, sagging, uneven texture and decreased elasticity (2). It has been suggested that aged skin has a disrupted barrier function and altered permeability, resulting in a dry appearance and an enhanced risk of skin disorders $(3,4)$. A number of processes are also impaired in aged skin, including angiogenesis, lipid and sweat production, immune function and vitamin D synthesis. This often results in impaired wound healing, atrophy, vulnerability to external stimuli and in the development of several benign and malignant diseases (3). Aging of the skin is induced by both intrinsic and extrinsic factors $(5,6)$, all of which lead to a reduced structural integrity and the loss of physiological function (6). Critically, understanding the mechanisms of skin aging is necessary for developing improved skin care products that delay this process and reduce the hazardous effects of aging-inducing factors $(5,7)$.

Apigenin (4',5,7-trihydroxyflavone), a member of the flavone subclass of flavonoids, is widely found in herbs, fruits and vegetables, and thus, is a substantial component of the human diet. It has been shown to possess a variety of biological characteristics, including antioxidant (8) and anti-inflammatory properties (9). It has also been shown to exert tumor-inhibitory effects (10) and to promote neurogenesis (11). Apigenin has also been shown to enhance wound healing and tissue repair in the skin of diabetic rats (12). During the process of wound healing, fibroblasts secrete collagen, and the formation of collagen-rich granulation tissue is vital for the pathophysiological mechanisms of wound closure (13).

Ultraviolet (UV) radiation that reaches the earth's surface is comprised of wavelength ranges referred to as UVB $(280-315 \mathrm{~nm})$ and UVA (315-400 $\mathrm{nm}$ ); UV radiation within the UVC wave- 
band (100-280 nm) is absorbed entirely within the atmosphere. UV irradiation is the main culprit implicated in premature skin aging, which is referred to as photoaging. A number of studies have explored the relative contributions of UVA and UVB to the aging phenotype (14-16). Notably, while UVA photons are, on average, 1,000 times less energetic than UVB photons, they are capable of inducing aging-related changes even in the dermis, partly due to their greater average depth of skin penetration than UVB photons (17).

Photoaging is characterized by the macro- and microstructural deterioration of the skin, which includes damage to collagen fibers, the excessive deposition of abnormal elastic fibers and increased levels of glycosaminoglycans (18-20). Among these factors, matrix metalloproteinases (MMPs) are thought to play a major role in mediating UV-induced skin aging (17). The MMPs are a family of structurally related molecules, including collagenase-1 (MMP-1), collagenase-3 (MMP-13), gelatinases A and B (MMP-2 and MMP-9, respectively), stromelysin-1 (MMP-3), membrane-type MMPs, and others, all of which are capable of degrading components of the extracellular matrix, such as collagen, elastin, fibronectin, proteoglycans and laminin (21). In particular, MMP-1 initiates the degradation of type I and III fibrillary collagen, MMP-9 (gelatinase B) further degrades the collagen fragments produced, and MMP-3 (stromelysin-1) degrades type IV collagen and activates pro-MMP-1 (22-24).

In the present study, we examined the protective effects of apigenin on skin aging and demonstrated that apigenin induces anti-aging effects in skin by improving its barrier function and reducing UVA-induced damage.

\section{Materials and methods}

Cell culture. Normal human dermal fibroblasts (nHDFs; Lonza, Basel, Switzerland) were cultured in Dulbecco's modified Eagle's medium (DMEM; Gibco Life Technologies, Carlsbad, CA, USA), supplemented with $10 \%$ fetal bovine serum (FBS; Sigma-Aldrich, St. Louis, MO, USA) and 1\% penicillin/streptomycin (Gibco Life Technologies) at $37^{\circ} \mathrm{C}$ in an atmosphere of $5 \% \mathrm{CO}_{2}$. Apigenin was purchased from Sigma-Aldrich and dissolved in dimethyl sulfoxide (DMSO).

UVA irradiation. The $\mathrm{nHDFs}\left(1 \times 10^{6} /\right.$ well) were seeded into 6-well plates and cultured until 70-80\% confluent. Prior to irradiation, the cells were washed twice with phosphate-buffered saline (PBS). Fresh PBS was then added, and the cells were irradiated with UVA light ( $25 \mathrm{~J} / \mathrm{cm}^{2}$ UVA; UVA lamp; UVP, Inc., Upland, CA, USA). The radiation intensity was monitored by a fiber optic spectrometer system USB2000 (Ocean Optics, Dunedin, FL, USA). The control cells were treated identically, except for the exposure to UV light. Following irradiation, various concentrations $(0-100 \mu \mathrm{M})$ of the treatment agent (apigenin) in fresh medium were added to cells at $37^{\circ} \mathrm{C}$ for $24 \mathrm{~h}$.

Cell viability assay. The $\mathrm{nHDF}$ were seeded at a density of $3 \times 10^{3}$ cells/well in 96-well plates and incubated for $24 \mathrm{~h}$. The cells were irradiated with UVA $\left(0-50 \mathrm{~J} / \mathrm{cm}^{2}\right)$ and incubated with various concentrations of apigenin (0-200 $\mathrm{mM}$ ) for 24 h. nHDF cell toxicity due to apigenin was evaluated using the EZ-Cytox Cell Viability Assay kit (Itsbio, Seoul, Korea), a water-soluble tetrazolium salt (WST-1) assay. WST-1 solution was added to the cultured cells at a volume equal to $10 \%$ that of the culture medium, and the cells were then incubated at $37^{\circ} \mathrm{C}$ for $1 \mathrm{~h}$. Cell viability was evaluated by measuring the absorbance at $450 \mathrm{~nm}$ using an iMark microplate reader (Bio-Rad, Hercules, CA, USA ).

Isolation of total RNA and quantitative PCR. Total RNA was isolated using TRIzol reagent (Invitrogen Life Technologies, Carlsbad, CA, USA) according to the manufacturer's instructions. The purity and concentration of the RNA were evaluated using a MaestroNano ${ }^{\circledR}$, a microvolume spectrophotometer (Maestrogen, Las Vegas, NV, USA), and cDNAs were synthesized using the miScript II RT kit (Qiagen, Hilden, Germany) according to the manufacturer's instructions. In order to evaluate the expression of MMP-1, quantitative PCR was performed using the following primers: forward, 5'-TCT GACGTTGATCCCAGAGAGCAG-3' and reverse, 5'-CAGGG TGACACCAGTGACTGCAC-3' using EvaGreen dye (Solis BioDyne, Tartu, Estonia) with Line-Gene K software (Bioer Technology Co., Ltd., Hangzhou, China). The Ct value for each gene was normalized to $\beta$-actin using the following primers: forward, 5'-GGATTCCTATGTGGGCGACGA-3' and reverse, 5'-CGCTCGGTGAGGATCTTCATG-3'. The relative expression levels of each gene were calculated using the $2^{-\Delta \Delta \mathrm{Ct}}$ method, as previously described (25).

Senescence-associated (SA)- $\beta$-galactosidase assay. The expression of galactosidase as a marker for senescent nHDFs was determined using the SA- $\beta$-galactosidase staining kit (BioVision, Inc., Milpitas, CA, USA) following the manufacturer's instructions. The nHDFs were seeded at a density of $2 \times 10^{5}$ cells/well in $60 \mathrm{~mm}$ cell culture plates and incubated at $37^{\circ} \mathrm{C}$ until they were $90 \%$ confluent. The cells were then pre-treated with apigenin, irradiated with UVA, and incubated for $24 \mathrm{~h}$. These cells were washed with PBS and fixed by treatment with $0.5 \mathrm{ml}$ fixing solution/well (4\% formaldehyde, $0.5 \%$ glutaraldehyde in PBS buffer, $\mathrm{pH}$ 7.2) for $1 \mathrm{~h}$. The fixed cells were stained in staining solution mix (staining solution, $470 \mu \mathrm{l}$; staining supplement, $5 \mu \mathrm{l} ; 20 \mathrm{mg} / \mathrm{ml} \mathrm{X-Gal} \mathrm{in} \mathrm{dimethyl-}$ formamide, $25 \mu \mathrm{l}$ ) for $24 \mathrm{~h}$, at $37^{\circ} \mathrm{C}$. After 1 day, $70 \%$ glycerol ( $1 \mathrm{ml} /$ well) was added, and images were captured using an an Olympus IX51 microscope (Olympus, Tokyo, Japan).

Human subjects and clinical evaluation. All clinical evaluations were approved by the Ethics Committee of the Korea Institute for Skin and Clinical Sciences and performed in accordance with the Declaration of Helsinki Principles. We enrolled 40 women, aged over 30 years, in a randomized and double-blinded clinical trial. The subjects were selected based on age and were not pregnant or nursing. All subjects were informed about the objective of the study, signed an informed consent, and agreed to use only our products for skin care during the study duration. Factors for dropping out of the trial included itching, erythema, or hindrance to evaluation by excessive drinking or smoking. Th subjects were divided into the control and experimental groups, each containing 20 subjects (control group, 44.40 \pm 5.97 years; experimental group, $45.30 \pm 6.29$ years). All subjects were subjected to the same conditions, apart from the experimental group which 
was administered the test treatment. The study duration was 4 weeks, and no participants dropped out. Biometric parameters were measured 3 times: before application, and then at 2 and 4 weeks after application. An investigator also questioned the subjects about their condition and performed visual evaluations for skin disorders, such as erythema, itching, scaling, edema, tingling and burning sensations, at each visitation.

Experimental procedures. To investigate the effects of apigenin on dermal density, skin elasticity, skin texture, moisture, transepidermal water loss (TEWL) and fine wrinkles around the eyes (also known as crow's feet), the subjects were instructed to apply $2 \mathrm{~g}$ of the test treatment to the face, including the eye rim, every morning and night for 4 weeks. The subjects and investigators were blinded to the test and control treatments. At each visit, all subjects washed with the cleanser provided and lay quietly in a room with a constant temperature $\left(22 \pm 1^{\circ} \mathrm{C}\right)$ and humidity $(45 \pm 5 \%)$, so that they would all be evaluated under the same conditions. The cream provided to the experimental group contained $1 \%$ (wt $\%$ ) apigenin; whereas, the cream provided to the control group was prepared using the same volume of water in the place of apigenin.

Measurement of skin elasticity. To evaluate the improvement in skin elasticity, a DermaLab USB elasticity probe (Cortex Technology Inc., Hadsund, Denmark) was applied to the skin, and the results were analyzed using the associated application software, version 1.09. The measurement was performed by applying a single fixed elasticity probe on the left cheek of a subject. To analyze the measured value (in MPa), Young's modulus (E) was used, and the detected value is dependent on skin elasticity. To evaluate improvement, measurements were taken 3 times, before treatment and both at 2 and 4 weeks after application.

Measurement of dermal density. To evaluate dermal density, a DUB ${ }^{\circledR}$ SkinScanner (taberna pro medicum, Luneburg, Germany) was utilized. Dermal density was measured (in $\mu \mathrm{m}$ ) $3 \mathrm{~cm}$ beside the left eye, applying a couplant for ultrasonic examination. The analysis range was limited to the region between the dermis and the upper panniculus. To evaluate improvement, measurements were performed three times, before treatment and both 2 and 4 weeks after first application.

Measurement of length of crow's feet. To evaluate the improvement of wrinkles, particularly crow's feet, a Robo skin analyzer CS50 (Inforward Inc., Tokyo, Japan) was used. All facial images were captured under the same position and with equal lighting. The capturing was performed 3 times at each evaluation, on the front, left and right sides of the face. To evaluate improvement, measurements were performed 3 times, before treatment and both at 2 and 4 weeks after application. We analyzed the captured images matching the facial feature points to reenact accurately, and the measurement unit was in $\mathrm{mm}$.

Evaluation of skin moisture. To evaluate improvement in skin moisture, a DermaLab USB moisture probe (Cortex Technology Inc.) was applied to the skin, and the data were analyzed using the associated application software, version 1.09. All subjects were evaluated on the same region of the right cheek, 5 times consecutively, and we calculated the mean value, excluding the maximum and minimum values. To evaluate improvement, measurements were performed 3 times, before treatment and both at 2 and 4 weeks after application. The probe measures skin conductance in micro Siemens $(\mu \mathrm{S})$, and the numerical value is dependent on skin moisture.

Measurements of TEWL. To evaluate improvements in TEWL, a DermaLab USB TEWL probe (Cortex Technology, Inc.) was applied to the skin, and the data were analyzed using the associated application software, version 1.09. The measurement was performed 5 times consecutively, on the right cheek of the subjects, and we calculated the mean value, excluding the maximum and minimum values. To evaluate improvement, measurements were performed 3 times, before treatment and both at 2 and 4 weeks after application.

Measurements of facial evenness. To evaluate improvements in facial evenness, a PRIMOS Lite system (field of view 45x30; GFMesstechnik GmbH, Teltow, Germany) was used, and the captured clinical images were analyzed using the associated imaging software, PRIMOS Lite version 5.6E. The images were captured 3 times consecutively, on the left side of the forehead of the subjects. We analyzed facial evenness by calculating surface roughness, Ra (average of all heights and epths to the reference plane) value. The Ra value, which is the most well used measurement for facial evenness, is the arithmetic mean of the absolute values within the total measurement range. To evaluate improvement, measurements were performed 3 times, before treatment and both at 2 and 4 weeks after application.

Statistical analysis. For cellular efficacy tests, all results are presented as the mean percentage \pm standard deviation (SD) of 3 independent experiments. Differences with a P-value $<0.05$, as determined by the Student's t-test, were considered statistically significant. For clinical efficacy tests, statistical analyses were conducted using SPSS software (SPSS, version 17.0 for Windows; IBM SPSS, Armonk, NY, USA). Paired Student's $\mathrm{t}$-tests were performed in the cases of repeated measurements on the same subject. To analyze subject questionnaires, the mean values, standard deviations and percentages were calculated. The formula used to measure the percentage change for each skin parameter was 'Percentage change $=[(\mathrm{A}-\mathrm{B}) / \mathrm{B}] \mathrm{x} 100$ ', where $\mathrm{A}$ is defined as the individual value of any parameter at the 2-and 4-week visits, and B represents the zero hour of the assessed parameter.

\section{Results}

Cytotoxicity of apigenin and UVA in human dermal fibroblasts. To determine whether apigenin affects nHDF viability, the cells were exposed to apigenin at concentrations ranging from $0-100 \mu \mathrm{M}$ for $24 \mathrm{~h}$. As shown in Fig. 1, apigenin reduced cell viability by $1.23 \%$ at $1 \mu \mathrm{M}, 3.63 \%$ at $5 \mu \mathrm{M}$, and $12.61 \%$ at $20 \mu \mathrm{M}$. The apigenin-induced cytotoxicity increased significantly at concentrations $>50 \mu \mathrm{M}$. Therefore, we used the concentration of $20 \mu \mathrm{M}$ as the maximum concentration in all the subsequent experiments.

To evaluate the effects of apigenin on the viability of damaged cells, the nHDFs were irradiated with $25 \mathrm{~J} / \mathrm{cm}^{2} \mathrm{UVA}$, 


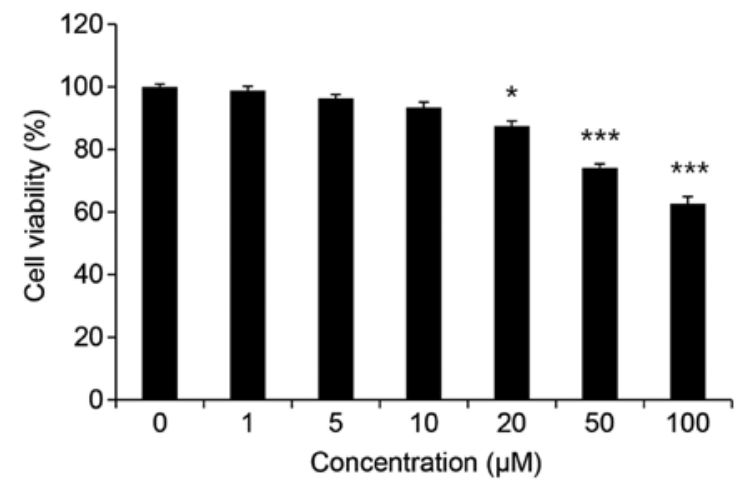

Figure 1. Cytotoxicity of apigenin in normal human dermal fibroblasts (nHDFs) nHDFs were treated with apigenin at the indicated concentrations for $24 \mathrm{~h}$. The results are representative of 3 independent experiments (means \pm SD are shown). ${ }^{*} \mathrm{P}<0.05$ and ${ }^{* * *} \mathrm{P}<0.001$, as determined by the Student's t-test.

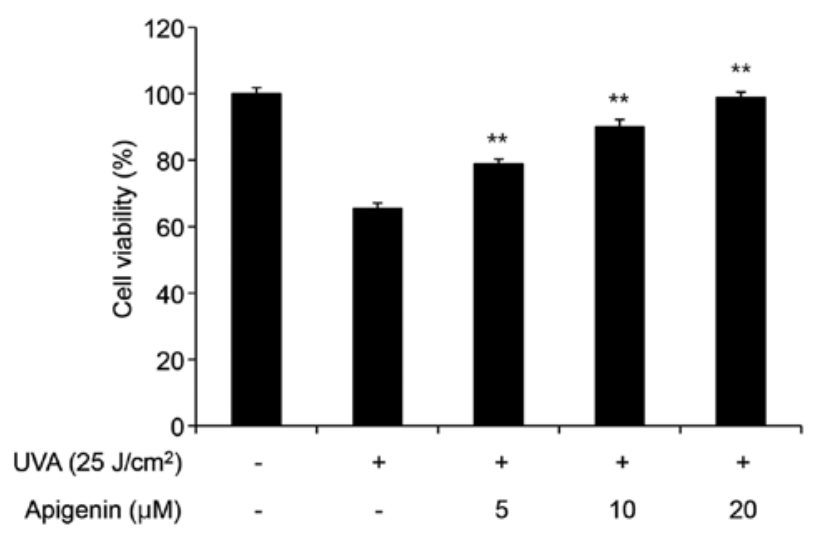

Figure 2. Effect of apigenin on the viability of UVA-irradiated normal human dermal fibroblasts (nHDFs). nHDFs were irradiated with $25 \mathrm{~J} / \mathrm{cm}^{2}$ UVA and then post-treated with apigenin for $24 \mathrm{~h}$. The results are representative of 3 independent experiments (means $\pm \mathrm{SD}$ are shown). ${ }^{* *} \mathrm{P}<0.01$, as determined by the Student's t-test.

and these cells were then treated with apigenin at various concentrations. As shown in Fig. 2, this dose of UVA reduced cell viability by $34.60 \%$; however, treatment with 10 and $20 \mu \mathrm{M}$ apigenin increased viability back to 90.00 and $98.92 \%$, respectively, suggesting that apigenin protects cells from UVA-induced cytotoxicity.

Senescent cell detection assay. We then investigated the ability of apigenin to inhibit senescence, using a SA- $\beta$-galactosidase assay. When the cells were irradiated with $25 \mathrm{~J} / \mathrm{cm}^{2} \mathrm{UVA}$, the percentage of senescent cells was found to be as high as $61.29 \%$. This number decreased in a dose-dependent manner to 50.49, 32.03 and $17.34 \%$ when cells were post-treated with 5,10 and $20 \mu \mathrm{M}$ apigenin, respectively (Fig. 3). These results indicate that UVA acts as a stimulator of senescence, and that apigenin can inhibit UVA-induced cellular senescence.

Analysis of MMP-1 mRNA expression. UVA radiation corresponds to $90-95 \%$ of solar UV radiation (26) and is mainly responsible for the high production of reactive oxygen species (ROS) in skin, leading to oxidative stress (27). ROS are able to induce several disruptive cellular processes, such as senescence, DNA cleavage, lipid peroxidation and cell

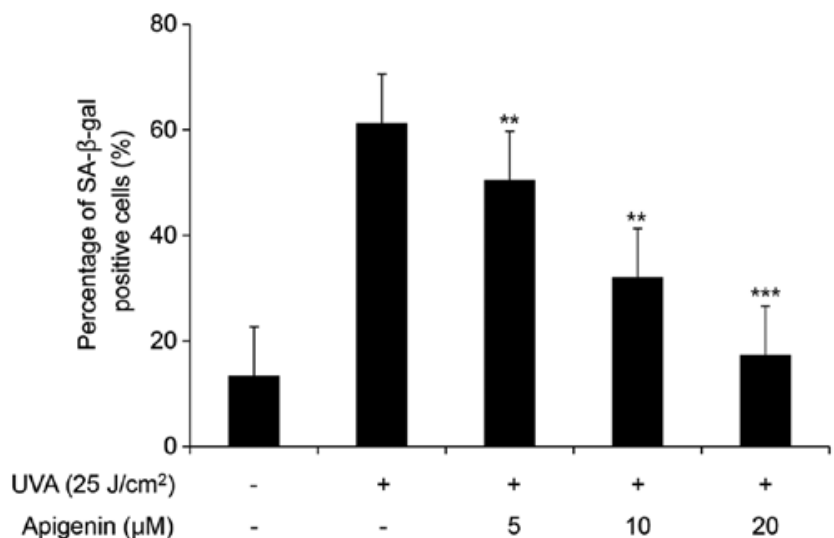

Figure 3. Inhibitory effect of apigenin on cellular senescence in UVA-irradiated normal human dermal fibroblasts (nHDFs). nHDFs were irradiated with $25 \mathrm{~J} /$ $\mathrm{cm}^{2}$ UVA and then post-treated with apigenin for $24 \mathrm{~h}$. Senescent nHDFs were detected using an optical microscope. The results are representative of 3 independent experiments (means $\pm \mathrm{SD}$ are shown). ${ }^{* *} \mathrm{P}<0.01$ and ${ }^{* *} \mathrm{P}<0.001$, as determined by the Student's t-test.

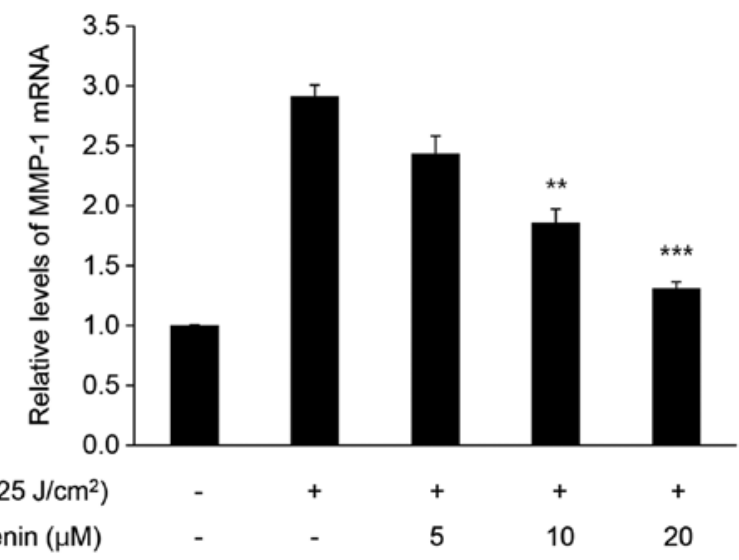

Figure 4. Effect of apigenin on matrix metalloproteinase (MMP-1) mRNA expression in normal human dermal fibroblasts (nHDFs). Relative mRNA expression levels of MMP-1 in UVA- and apigenin-treated HDFs, as measured by quantitative PCR. The results are representative of 3 independent experiments (means \pm SD are shown). ${ }^{* *} \mathrm{P}<0.01$ and ${ }^{* * * *} \mathrm{P}<0.001$, as determined by the Student's t-test.

death (28). In addition, UVA induces the expression of MMP-1 in dermal fibroblasts in vivo and stimulates the expression of MMP-1, MMP-2 and MMP-3 in cell culture, all of which are induced during wrinkling and skin aging (29,30). MMP-1, when generated from fibroblasts, has been reported to ultimately promote a decrease in collagen $(23,31,32)$. Collagen is the most abundant protein in the dermis, and type- 1 collagen, in particular, provides structure to skin and composes $>90 \%$ of collagen in the body (33). We found that the mRNA expression of MMP-1 in the UVA-irradiated nHDFs increased up to 2.91-fold as compared with the non-irradiated cells. However, treatment with 5, 10 and $20 \mu \mathrm{M}$ apigenin reduced MMP-1 expression 2.43-, 1.85- and 1.31-fold, respectively (Fig. 4), indicating that apigenin inhibits the UVA-induced induction of MMP-1 expression.

Evaluation of dermal density. To evaluate the effects of apigenin on skin aging in vivo, we measured the density of the 


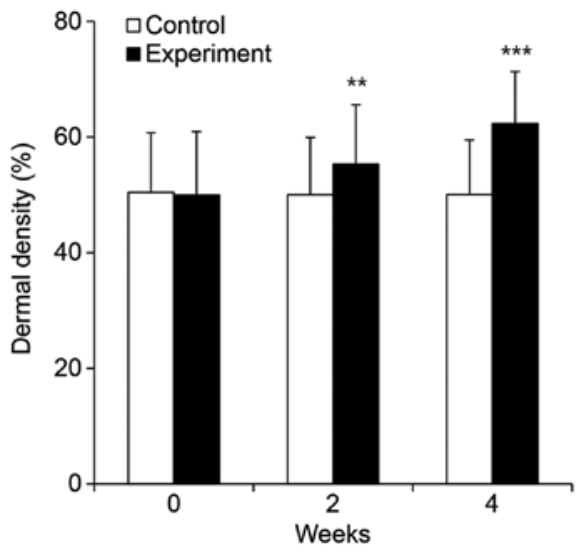

Figure 5. Dermal density of subjects treated with apigenin-containing cream or the control cream. The measurements were performed 3 times, namely, before application and after 2 and 4 weeks of use, using a DUB-Skin scanner, $3 \mathrm{~cm}$ beside the left eye, applying the couplant for ultrasonic examination. The unit of measurement is micro Meter $(\mu \mathrm{m}) .{ }^{* *} \mathrm{P}<0.01$ and ${ }^{* * * *} \mathrm{P}<0.001$, as determined by the Student's t-test.

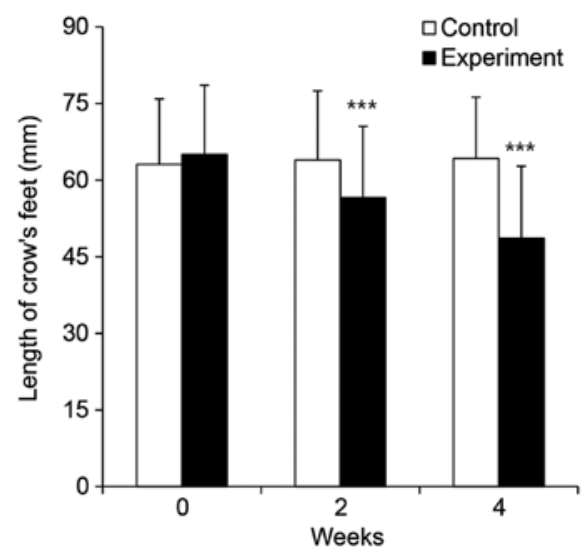

Figure 6. Length of crow's feet in subjects treated with the apigenin-containing cream or the control cream. The measurements were performed 3 times, namely, before application and after 2 and 4 weeks of use, using a Robo skin analyzer CS50, on the front, left, and right side of face. ${ }^{* * *} \mathrm{P}<0.001$ as determined by the Student's t-test.

dermis in subjects treated with a cream containing $1 \%$ apigenin. Using a DUB SkinScanner, we found that the subjects using the non-apigenin-containing control cream displayed a mean density of $50.41 \mu \mathrm{m}$ before use, and densities of 50.02 and $50.05 \mu \mathrm{m}$ after 2 and 4 weeks, respectively (Fig. 5). However, subjects using the apigenin-containing cream displayed a mean density of $49.96 \mu \mathrm{m}$ before use, and densities of 55.31 and 62.32 after 2 and 4 weeks of application, respectively (Fig. 5). Dermal density measurement, represented as a mathematical value, is proportional to density, and these experimental data were statistically significant $(\mathrm{P}<0.001)$. To compare the results from the treatment and control groups, we calculated the improvement as a percentage based on the density values before and after application. Using this metric, the dermal density improvement was found to be -0.77 and $-0.72 \%$ after 2 and 4 weeks of application, respectively, in the control group. Conversely, in the experimental group, the dermal density improvement was calculated as 10.70 and $24.75 \%$ after 2 and

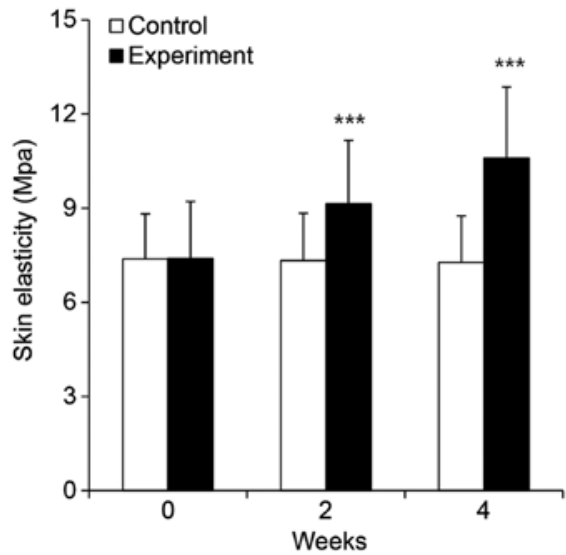

Figure 7. Skin elasticity in subjects treated with the apigenin-containing cream or the control cream. Skin elasticity measurements were performed 3 times, namely, before application and after 2 and 4 weeks of use, using a DermaLab USB elasticity probe. The detection was on the left cheek of subject, and the data were analyzed using application software, version 1.09 . ${ }^{* * *} \mathrm{P}<0.001$ as determined by the Student's t-test.

4 weeks of application, respectively. These results suggest that the topical application of apigenin enhances dermal thickness.

Evaluation of crow's feet length. We then measured the length of crow's feet in the subjects treated with the apigenin-containing cream and the controls. In the control group, the mean length was found to be $63.10 \mathrm{~mm}$ before application, and 63.95 and $64.25 \mathrm{~mm}$ after 2 and 4 weeks of application, respectively (Fig. 6). In the experimental group, the mean length was $65.05 \mathrm{~mm}$ prior to application, and 56.60 and $48.65 \mathrm{~mm}$ after 2 and 4 weeks of application, respectively (Fig. 6). The measured values for the experimental group were statistically significant $(\mathrm{P}<0.001)$. To compare the results from the control and experimental groups, we calculated the improvement as a percentage based on the values before and after application. For the control group, the percentage improvement was calculated as -1.35 and $-1.82 \%$ after 2 and 4 weeks of application, respectively. Whereas for the experimental group, the percentage improvement was found to be 12.99 and $25.21 \%$ after 2 and 4 weeks of application, respectively. These data suggest that the topical application of apigenin can lead to a reduction in wrinkle length.

Evaluation of skin elasticity. The dermis is composed of an extracellular matrix consisting of fibrous proteins, such as collagen and elastin, and is involved in the regulation of skin elasticity. Factors such as ROS, UV, or age can cause skin damage, wrinkle formation and a reduction in elasticity through the estructural denaturation of collagen and elastin (34). In order to examine the effects of apigenin on skin elasticity, we measured elasticity in subjects treated with apigenin-containing cream or the control cream using a DermaLab USB probe. In the control group, elasticity was found to be $7.38 \mathrm{MPa}$ before application, and 7.33 and 7.27 MPA after 2 and 4 weeks of application, respectively. In the experimental group, elasticity was 7.40 MPa before application, and 9.14 and $10.60 \mathrm{MPa}$ after 2 and 4 weeks of application, respectively (Fig. 7). The experimental group values were statistically significant $(\mathrm{P}<0.001)$. To compare the results from the control and experimental groups, we 


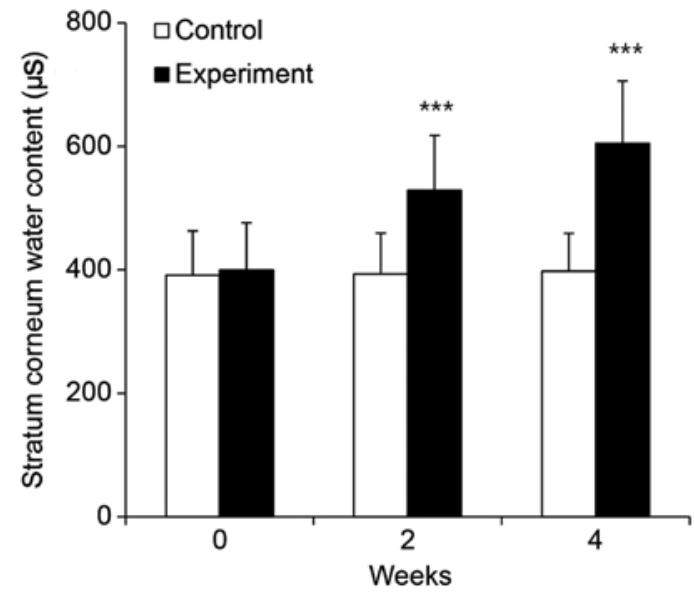

Figure 8. Skin moisture content in subjects treated with the apigenin-containing cream or the control cream. Measurements were taken 3 times, namely, before application and after 2 and 4 weeks of use. A DermaLab USB moisture probe was applied, and the data were analyzed using the associated software application version 1.09. ${ }^{* * *} \mathrm{P}<0.001$ as determined by the Student's t-test.

calculated the improvement as a percentage based on the values before and after application. For the control group, the percentage improvement was calculated to be -0.68 and $-1.42 \%$ after 2 and 4 weeks of application, respectively. However, the experimental group showed an improvement of 23.60 and $43.34 \%$ after 2 and 4 weeks of treatment, respectively. These results suggest that the topical applicatino of apigenin increases skin elasticity.

Apigenin-containing cream improves skin hydration. Keratinocyte moisture content is pivotal for maintaining moisture in the skin. Normal keratinocytes maintain 10-30\% moisture; however, when the moisture content drops below $10 \%$, keratinocytes are unable to maintain the skin's barrier function, and skin becomes dry, acquires an uneven texture and produces wrinkles, accelerating senescence (4). To evaluate the effect of apigenin treatment on skin moisture, we analyzed the skin moisture content in our study subjects using the DermaLab USB moisture probe. We found that the moisture content in the control group was $391.25 \mu \mathrm{S}$ before use, and 393.30 and $397.87 \mu \mathrm{S}$ after 2 and 4 weeks of application, respectively (Fig. 8). By contrast, the moisture content in the experimental group, which used the apigenin-containing cream, was $399.48 \mu \mathrm{S}$ before use, and 528.75 and $604.74 \mu \mathrm{S}$ after 2 and 4 weeks of application, respectively (Fig. 8). To compare the results from the control and experimental groups, we calculated the degree of improvement as a percentage based on the values before and after application. For the control group, moisture was increased by 0.52 and $1.69 \%$ after 2 and 4 weeks, respectively. These changes were not statistically significant $(\mathrm{P}>0.05)$, indicating that the control cream had no measurable effect on moisture content. Conversely, the use of the apigenin-containing cream significantly improved skin moisture by 32.36 and $51.38 \%$ after 2 and 4 weeks, respectively $(\mathrm{P}<0.001)$. These data demonstrate that use of apigenin-containing cream results in an improved skin moisture content.

Apigenin-containing cream improves TEWL. To determine the efficacy of apigenin as a skin moisturizer, we used the

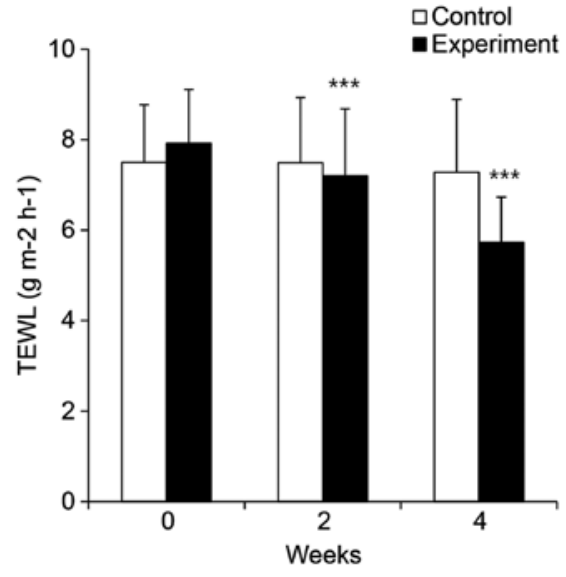

Figure 9. Transepidermal water loss (TEWL) in subjects treated with the apigenin-containing cream or control cream. Measurements were performed 3 times, namely, before application and after 2 and 4 weeks of use, using a DermaLab USB TEWL probe, and the data were analyzed using the associated application software, version $1.09 .{ }^{* * *} \mathrm{P}<0.001$ as determined by the the Student's t-test.

DermaLab USB TEWL probe to measure TEWL in the skin of subjects who used either the control or apigenin-containing cream. In the control subjects, the TEWL was found to be $7.50 \mathrm{~g} \mathrm{~m}^{-2} \mathrm{~h}^{-1}$ before use, and 7.49 and $7.28 \mathrm{~g} \mathrm{~m}^{-2} \mathrm{~h}^{-1}$ after 2 and 4 weeks of application, respectively (Fig. 9). By contrast, in the experimental group, the TEWL was $7.92 \mathrm{~g} \mathrm{~m}^{-2} \mathrm{~h}^{-1}$ before use, and 7.20 and $5.73 \mathrm{~g} \mathrm{~m}^{-2} \mathrm{~h}^{-1}$ after 2 and 4 weeks of application, respectively (Fig. 9). To compare the results from the control and experimental groups, we calculated the improvement in TEWL as a percentage based on the values before application. In the control group, TEWL was increased by 0.20 and $2.93 \%$ after 2 and 4 weeks of use, respectively. These changes were not statistically significant $(\mathrm{P}>0.05)$, indicating that the control cream had no measurable effect on TEWL. Conversely, the use of the apigenin-containing cream significantly improved the TEWL by 9.10 and $27.61 \%$ after 2 and 4 weeks, respectively $(\mathrm{p}<0.001)$. Through these experiments, we identified an improvement in TEWL as an outcome of using the apigenin-containing cream.

Use of apigenin-containing cream improves the evenness of skin texture. The thickness of the stratum corneum changes depending on its moisture content, and insufficient moisture in this layer gradually roughens skin texture (35). To investigate the effects of the apigenin-containing cream on skin texture, facial skin evenness was measured using a PRIMOS Lite system. Evenness in the control group was $19.71 \mathrm{Ra}$ before use, and 19.43 and 19.30 Ra after 2 and 4 weeks of application, respectively (Fig. 10). By contrast, skin evenness in the experimental group was $20.05 \mathrm{Ra}$ before use, and 18.41 and $16.11 \mathrm{Ra}$ after 2 and 4 weeks of application, respectively (Fig. 10). To compare the results from the control and experimental groups, we calculated the improvement as a percentage based on the value before application. Consequently, we found that skin texture in the control group improved by 1.42 and $2.06 \%$, after 2 and 4 weeks of use, respectively. These data were not statistically significant $(\mathrm{P}>0.05)$, indicating that the control cream had no measurable effect on skin texture. However, the use of the apigenin-containing cream significantly improved 


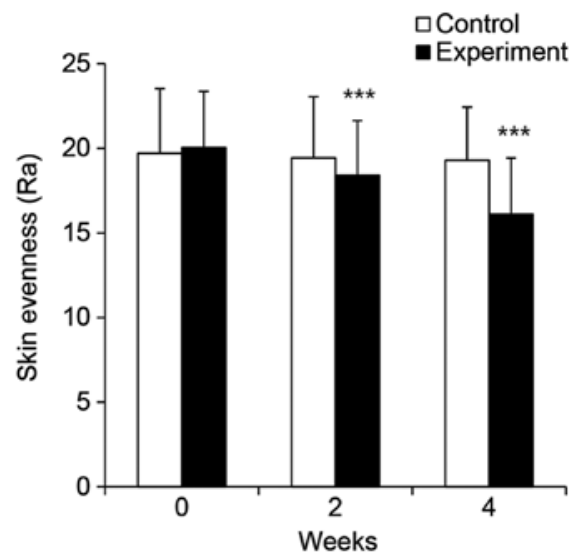

Figure 10. Skin evenness in subjects treated with the apigenin-containing cream or the control cream. Evenness of the skin surface was measured using the PRIMOS Lite system. Measurements were taken 3 times, namely, before application and after 2 and 4 weeks of use. The captured images were analyzed using the associated imaging software PRIMOS Lite version 5.6E. ${ }^{* * * *} \mathrm{P}<0.001$ as determined by the Student's t-test.

Table I. Adverse skin reactions reported by the subjects.

Abnormal reaction Severity Abnormal reaction Severity

\begin{tabular}{llll}
\hline Erythema & $0^{\mathrm{a}}$ & Tingling & 0 \\
Swelling (edema) & 0 & Burning & 0 \\
Scaling (epidermis) & 0 & Tightness & 0 \\
Itching & 0 & Prickling & 0 \\
\hline
\end{tabular}

${ }^{\mathrm{a}} 0$, none; 1 , mild; 2 , severe; 3 , very severe.

the evenness of skin texture by 8.20 and $19.65 \%$ after 2 and 4 weeks of application $(\mathrm{P}<0.001)$, respectively, suggesting that the use of the apigenin-containing cream can improve skin texture.

Analysis of adverse effects of apigenin-containing cream. In this study, investigators questioned the subjects individually about the condition of their skin and performed a visual evaluation of skin reactions, including erythema, itching, scaling, tingling, tightness, prickling and burning sensations at each visit. No extraordinary reactions were reported based on either visual evaluation or the questionnaire (Table I).

\section{Discussion}

Apigenin has been reported to have various biological activities in various cell types, such as antioxidant, anti-inflammatory, anti-mutagenic and anti-tumorigenic properties (36-39). However, it has not been determined whether apigenin can affect the aging process. In this study, we examined the effects of apigenin, particularly with regard to skin aging and wrinkling, using cellular and clinical efficacy experiments.

We first evaluated the viability of nHDFs that were irradiated with $25 \mathrm{~J} / \mathrm{cm}^{2}$ UVA and found that the post-treatment of UVA-irradiated nHDFs with apigenin significantly reduced cell cytotoxicity. This suggests that apigenin can reduce and/or mitigate UVA-induced cellular damage. We then evaluated the effects of apigenin on cellular senescence using a SA- $\beta$ galactosidase assay and found that while the percentage of senescent nHDFs increased in response to UVA irradiation, treatment with apigenin reduced the percentage of senescent cells in a dose-dependent manner. Furthermore, under the same conditions, the mRNA expression of MMP-1 collagenase-1, which is a reported initiator for the degradation of type I and III fibrillary collagen and is induced in response to UVA irradiation, was reduced by apigenin in a dose-dependent manner (17). Thus, we demonstrated the cellular efficacy of apigenin in inhibiting UVA-induced growth arrest, cellular senescence and the expression of MMP-1 in nHDFs.

UVA, a component of the UV spectrum, has been reported to have a greater average skin penetration than other UV types. Based on previous studies $(17,29)$, UV irradiation, including UVA, is the main cause of skin aging, referred to as photoaging. It has been reported that collagen fibers, which are dermal components that maintain skin elasticity, are fragmented in photoaging skin (40). Furthermore, UV irradiation was found to decrease collagen synthesis and increase collagenolytic MMP synthesis in dermal fibroblasts $(22,23,41)$, which are believed to contribute to the furrows observed in photoaging skin. MMPs are capable of degrading all components of the extracellular matrix and are upregulated by UV.

Based on the results of our cellular experiments demonstrating an apigenin-mediated protection from UVA-induced toxicity and an inhibition of MMP-1 upregulation in $\mathrm{nHDFs}$, we constructed an apigenin-containing cream and a non-apigenin-containing control and enrolled 40 women ( $>30$ years old) in a randomized and doubleblinded clinical trial to examine the effects of apigenin on aging skin in vivo. We then evaluated a number of features associated with aging in the skin of subjects who used either the apigenin-containing cream or the control. Of note, we detected a significant improvement in dermal density and skin elasticity, and a reduction in the length of fine wrinkles, particularly crow's feet, after 2 and 4 weeks of application in the subjects using the apigenin-containing cream. These results indicate that the topical application of apigenin reduces aging phenomena and its aging-associated clinical signs.

The skin is an important organ that separates the human body from the external environment. It has been previously reported that both the barrier function and the water-holding capacity of human skin are decreased by solar UV exposure (42). Therefore, the dryness of photoaging skin cannot be explained only under the direct influence of UV irradiation to skin cells. Therefore, we also measured skin moisture content, TEWL, and skin texture in subjects treated with apigenin-containing cream or the control. We found that all these parameters were significantly improved after 2 and 4 weeks of use in those who applied the apigenin-containing cream. These results strongly suggest that the topical application of the apigenin cream can improve aging skin, by enhancing the skin's barrier function.

\section{Acknowledgements}

This study was supported by the KU Research Professor Program (H.-J. Cha) of Konkuk University. Support was also provided by grants from the Ministry of Science, ICT 
and Future Planning (grant no. 20110028646), the Korean Health Technology R\&D Project, the Ministry of Health \& Welfare (grant no. HN13C0075), and the Ministry of Oceans and Fisheries, Republic of Korea (grant no. OF123321).

\section{References}

1. Viña J, Borrás $\mathrm{C}$ and Miquel $\mathrm{J}$ : Theories of ageing. IUBMB Life 59: 249-254, 2007.

2. Jenkins G: Molecular mechanisms of skin ageing. Mech Ageing Dev 123: 801-810, 2002.

3. Zouboulis CC and Makrantonaki E: Clinical aspects and molecular diagnostics of skin aging. Clin Dermatol 29: 3-14, 2011.

4. Hashizume H: Skin aging and dry skin. J Dermatol 31: 603-609, 2004.

5. Farage MA, Miller KW, Elsner P and Maibach HI: Intrinsic and extrinsic factors in skin ageing: a review. Int J Cosmet Sci 30: 87-95, 2008.

6. Landau M: Exogenous factors in skin aging. Curr Probl Dermatol 35: 1-13, 2007.

7. Elsner P, Fluhr JW, Gehring W, Kerscher MJ, Krutmann J, Lademann J, Makrantonaki E, Wilhelm KP and Zouboulis CC: Anti-aging data and support claims - consensus statement. J Dtsch Dermatol Ges 9 (Suppl 3), S1-32, 2011.

8. Sharma H, Kanwal R, Bhaskaran N and Gupta S: Plant flavone apigenin binds to nucleic acid bases and reduces oxidative DNA damage in prostate epithelial cells. PLoS One 9: e91588, 2014.

9. Wang J, Liu YT, Xiao L, Zhu L, Wang Q and Yan T: Anti-inflammatory effects of apigenin in lipopolysaccharide-induced inflammatory in acute lung injury by suppressing COX-2 and NF-kB pathway. Inflammation 37: 2085-2090, 2014.

10. Polier G, Giaisi M, Köhler R, Müller WW, Lutz C, Buss EC, Krammer $\mathrm{PH}$ and Li-Weber M: Targeting CDK9 by wogonin and related natural flavones potentiates the anti-cancer efficacy of the Bcl-2 family inhibitor ABT-263. Int J Cancer 136: 688-698, 2015.

11. Taupin P: Apigenin and related compounds stimulate adult neurogenesis. Mars, Inc., the Salk Institute for Biological Studies: WO2008147483. Expert Opin Ther Pat 19: 523-527, 2009.

12. Lodhi S and Singhai AK: Wound healing effect of flavonoid rich fraction and luteolin isolated from Martynia annua Linn. on streptozotocin induced diabetic rats. Asian Pac J Trop Med 6 : 253-259, 2013.

13. Singer AJ and Clark RA: Cutaneous wound healing. N Engl J Med 341: 738-746, 1999.

14. Gonzaga ER: Role of UV light in photodamage, skin aging, and skin cancer: importance of photoprotection. Am J Clin Dermatol 10 (Suppl 1): 19-24, 2009.

15. Ham SA, Kang ES, Lee H, Hwang JS, Yoo T, Paek KS, Park C, Kim JH, Lim DS and Seo HG: PPAR inhibits UVB-induced secretion of MMP-1 through MKP-7-mediated suppression of JNK signaling. J Invest Dermatol 133: 2593-2600, 2013.

16. Lee YK, Cha HJ, Hong M, Yoon Y, Lee H and An S: Role of NF- $\mathrm{B}$-p53 crosstalk in ultraviolet A-induced cell death and G1 arrest in human dermal fibroblasts. Arch Dermatol Res 304: 73-79, 2012.

17. Mac-Mary S, Sainthillier JM, Jeudy A, Sladen C, Williams C, Bell $\mathrm{M}$ and Humbert P: Assessment of cumulative exposure to UVA through the study of asymmetrical facial skin aging. Clin Interv Aging 5: 277-284, 2010.

18. Smith JG Jr, Davidson EA, Sams WM Jr and Clark RD: Alterations in human dermal connective tissue with age and chronic sun damage. J Invest Dermatol 39: 347-350, 1962.

19. Grewe M, Trefzer U, Ballhorn A, Gyufko K, Henninger H and Krutmann J: Analysis of the mechanism of ultraviolet (UV) B radiation-induced prostaglandin E2 synthesis by human epidermoid carcinoma cells. J Invest Dermatol 101: 528-531, 1993.

20. Chung JH, Seo JY, Choi HR, Lee MK, Youn CS, Rhie G, Cho KH, Kim KH, Park KC and Eun HC: Modulation of skin collagen metabolism in aged and photoaged human skin in vivo. J Invest Dermatol 117: 1218-1224, 2001.

21. Inomata S, Matsunaga Y, Amano S, Takada K, Kobayashi K, Tsunenaga M, Nishiyama T, Kohno Y and Fukuda M: Possible involvement of gelatinases in basement membrane damage and wrinkle formation in chronically ultraviolet B-exposed hairless mouse. J Invest Dermatol 120: 128-134, 2003.

22. Fisher GJ, Datta SC, Talwar HS, Wang ZQ, Varani J, Kang S and Voorhees JJ: Molecular basis of sun-induced premature skin ageing and retinoid antagonism. Nature 379: 335-339, 1996.
23. Fisher GJ, Wang ZQ, Datta SC, Varani J, Kang S and Voorhees JJ: Pathophysiology of premature skin aging induced by ultraviolet light. N Engl J Med 337: 1419-1428, 1997.

24. Fisher GJ, Talwar HS, Lin J, Lin P, McPhillips F, Wang Z, Li X, Wan Y, Kang S and Voorhees JJ: Retinoic acid inhibits induction of c-Jun protein by ultraviolet radiation that occurs subsequent to activation of mitogen-activated protein kinase pathways in human skin in vivo. J Clin Invest 101: 1432-1440, 1998.

25. Livak KJ and Schmittgen TD: Analysis of relative gene expression data using real-time quantitative PCR and the 2(-Delta Delta C(T)) Method. Methods 25: 402-408, 2001.

26. Nichols JA and Katiyar SK: Skin photoprotection by natural polyphenols: Anti-inflammatory, antioxidant and DNA repair mechanisms. Arch Dermatol Res 302: 71-83, 2010.

27. Pandel R, Poljšak B, Godic A and Dahmane R: Skin photoaging and the role of antioxidants in its prevention. ISRN Dermatol 930164: 2013, 2013.

28. Tedesco AC, Martínez L and González S: Photochemistry and photobiology of actinic erythema: Defensive and reparative cutaneous mechanisms. Braz J Med Biol Res 30: 561-575, 1997.

29. Scharffetter K, Wlaschek M, Hogg A, Bolsen K, Schothorst A, Goerz G, Krieg T and Plewig G: UVA irradiation induces collagenase in human dermal fibroblasts in vitro and in vivo. Arch Dermatol Res 283: 506-511, 1991.

30. Chung JH, Seo JY, Lee MK, Eun HC, Lee JH, Kang S, Fisher GJ and Voorhees JJ: Ultraviolet modulation of human macrophage metalloelastase in human skin in vivo. J Invest Dermatol 119: 507-512, 2002.

31. Burke EM, Horton WE, Pearson JD, Crow MT and Martin GR: Altered transcriptional regulation of human interstitial collagenase in cultured skin fibroblasts from older donors. Exp Gerontol 29: 37-53, 1994.

32. Vincenti MP, White LA, Schroen DJ, Benbow U and Brinckerhoff CE: Regulating expression of the gene for matrix metalloproteinase-1 (collagenase): mechanisms that control enzyme activity, transcription, and mRNA stability. Crit Rev Eukaryot Gene Expr 6: 391-411, 1996.

33. Lim JY, Kim OK, Lee J, Lee MJ, Kang N and Hwang JK: Protective effect of the standardized green tea seed extract on UVB-induced skin photoaging in hairless mice. Nutr Res Pract 8: 398-403, 2014.

34. Kimura Y and Sumiyoshi M: Olive leaf extract and its main component oleuropein prevent chronic ultraviolet B radiation-induced skin damage and carcinogenesis in hairless mice. J Nutr 139: 2079-2086, 2009.

35. Tagami H, Ohi M, Iwatsuki K, Kanamaru Y, Yamada M and Ichijo B: Evaluation of the skin surface hydration in vivo by electrical measurement. J Invest Dermatol 75: 500-507, 1980.

36. Singh JP, Selvendiran K, Banu SM, Padmavathi R and Sakthisekaran D: Protective role of Apigenin on the status of lipid peroxidation and antioxidant defense against hepatocarcinogenesis in Wistar albino rats. Phytomedicine 11: 309-314, 2004.

37. Ha SK, Lee P, Park JA, Oh HR, Lee SY, Park JH, Lee EH, Ryu JH, Lee KR and Kim SY: Apigenin inhibits the production of NO and PGE2 in microglia and inhibits neuronal cell death in a middle cerebral artery occlusion-induced focal ischemia mice model. Neurochem Int 52: 878-886, 2008.

38. Myhrstad MC, Carlsen H, Nordström O, Blomhoff R and Moskaug JØ: Flavonoids increase the intracellular glutathione level by transactivation of the gamma-glutamylcysteine synthetase catalytical subunit promoter. Free Radic Biol Med 32: 386-393, 2002.

39. Wei H, Tye L, Bresnick E and Birt DF: Inhibitory effect of apigenin, a plant flavonoid, on epidermal ornithine decarboxylase and skin tumor promotion in mice. Cancer Res 50: 499-502, 1990.

40. Fligiel SE, Varani J, Datta SC, Kang S, Fisher GJ and Voorhees JJ: Collagen degradation in aged/photodamaged skin in vivo and after exposure to matrix metalloproteinase-1 in vitro. J Invest Dermatol 120: 842-848, 2003.

41. Chu ML and Prockop D: Collagen: gene structure. In: Connective Tissue and Its Heritable Disorders. 2nd edition. Wiley-Liss, Inc, New York, NY, pp149-165, 1993.

42. Lim SH, Kim SM, Lee YW, Ahn KJ and Choe YB: Change of biophysical properties of the skin caused by ultraviolet radiation-induced photodamage in Koreans. Skin Res Technol 14: 93-102, 2008. 